\title{
A Preliminary Scoping Study of Federated Learning for the Internet of Medical Things
}

\author{
Arshad FARHAD ${ }^{\mathrm{a}, 1}$, Sandra I. WOOLLEY ${ }^{\mathrm{a}}$ and Peter ANDRAS ${ }^{\mathrm{a}}$ \\ ${ }^{a}$ Keele University, Staffordshire, $U K$
}

\begin{abstract}
This paper presents a scoping review of federated learning for the Internet of Medical Things (IoMT) and demonstrates the limited amount of research work in an area which has potential to improve patient care. Federated Learning and IoMTas standalone technologies - have already proved to be highly disruptive but there is a need for further research to apply federated learning to the IoMT.
\end{abstract}

Keywords. Federated Learning, Internet of Medical Things, IoMT.

\section{Introduction}

Federated learning [1,2] is a learning model which addresses the problem of data governance and privacy by training algorithms collaboratively without transferring the data to another location. The Internet of Things (loT) is a driver for the connected healthcare paradigm [3]. The collection of medical devices (wearables, wireless sensors, etc.) and applications that connect to healthcare IT systems through online computer network is known as Internet of Medical Things (IoMT). By enabling decentralized machine learning and avoiding the need to centrally compile patient data, federated learning has considerable potential for privacy and security in the IoMT. This paper reports the preliminary results of a scoping study that maps the literature relevant to Federated Learning and IoMT and identifies key concepts.

\section{Method}

A scoping review methodology was chosen to map the literature for the fields of Federated Learning and the IoMTs. A scoping review is defined by Arskey and O'Malley [4] as a study that aims 'to map rapidly the key concepts underpinning a research area and the main sources and types of evidence available, and can be undertaken as standalone projects in their own right, especially where an area is complex or has not been reviewed comprehensively before'.

The search strategy was adapted from Luo et al. [5] and the searches were conducted to identify relevant literature using the keywords 'Federated Learning', 'Internet of Medical Things', and 'IoMT. The searches were conducted using Google Scholar and were further filtered by year for the last 10 years, 2010 to 2020.

\footnotetext{
${ }^{1}$ Corresponding Author, Arshad Farhad; E-mail: a.farhad@keele.ac.uk.
} 


\section{Results}

We analyzed the Google Scholar search results after individual search to present an overview of existing literature in "Federated Learning", "Internet of Medical Things", "IoMT", "(IoMT OR "internet of medical things" OR ((IoT OR "internet of things") AND (health OR medical OR healthcare))) AND "federated learning". Overall, published research contents is shown in Figure 1.

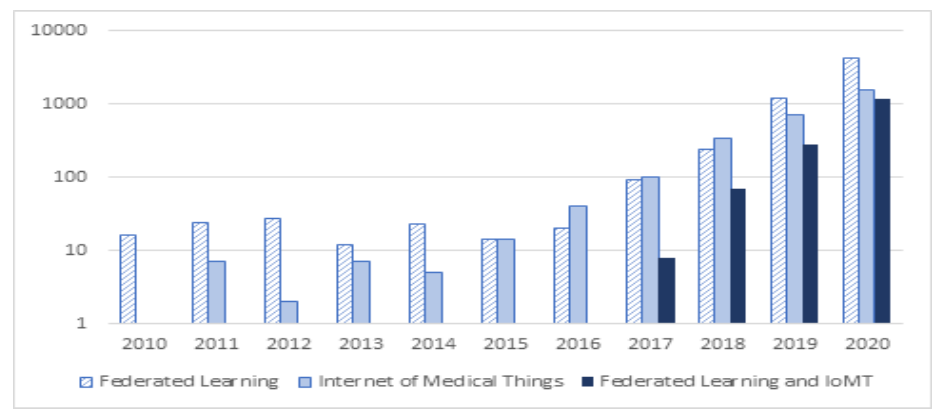

Figure 1. Search Results (Note: the vertical axis uses the logarithmic scale)

A total of 5813 articles were found on "Federated Learning" in the last ten years and almost $96 \%$ of which were published in the last three years. There are 2789 IoMT articles in the last 10 years, $97 \%$ of which were published in the last three years. However, only 1522 papers were found on "Federated Learning" AND "Internet of Medical Things"/" IoMT" OR "IOT/Internet of Things in healthcare and medical" all of which were all published in the last 4 years and the majority (1160 out of 1522) were published in the last 12 months.

\section{Discussion and Conclusion}

This preliminary scoping study of federated learning for the Internet of Medical Things has revealed an exponential increase in interest in recent years. However, the current work is limited, and further research is required to identify additional benefits of Federated Learning for IoMT.

\section{References}

[1] Yang Q, Liu Y, Chen T, Tong Y. Federated machine learning: concept and applications. ACM Trans. Intel. Syst. Technol. (TIST) 2019;10:12.

[2] McMahan B, Moore E, Ramage, D, Hampson S, y Arcas BA. Communication-efficient learning of deep networks from decentralized data. In Artificial Intelligence and Statistics, pp. 1273-1282, 2017

[3] Baker SB, Xiang W, Atkinson I. Internet of Things for Smart Healthcare: Technologies Challenges and Opportunities. IEEE Access. 2017;5: 26521-26544.

[4] Arksey H, O'Malley L. Scoping studies: towards a methodological framework. International Journal of Social Research Methodology. 2005;8:19-32.

[5] Luo J, Wu M, Gopukumar D, Zhao I. Big data application in biomedical research and health care: a literature review. Biomedical Informatics Insights. 2016;8: 1-10. 